\title{
Studies of Suspicious Lesions through Local Texture Analysis in Spine Radiographs
}

\author{
Richa Sharma \\ Research Associate \\ CERSSE, Jain University, \\ No. 52, Bellary Road Hebbal, \\ Bangalore-560024, India
}

\author{
T. R. Gopalakrishnan Nair \\ Director \\ Center for Advanced Technology Research, \\ RR Group Institutions, No. 14, \\ Ramohalli Cross, Kumbalgodu, \\ Bengalore-560074, India
}

\begin{abstract}
This paper presents a method to assess and classify the textural abnormalities created by the cellular changes made available through digital radiographs. In this process, digital $\mathrm{X}$-ray images of the spine were subjected to investigations. A study has been made to choose the optimum set of features selectively to train the classifier.

In most common applications, the feature vector is extracted from the image data using pixel intensity and magnitude of the frequency displayed as an image. In this case, the introduction of the phase angle of the frequency involved in generating the hue of the suspicious lesions considerably enhanced the success rate of the detection (approximately by $25 \%)$.

Our experimental study based on 32 subjects indicates that the proposed system is successfully classifying the abnormal regions with $94.37 \%$ mean Correct Classification Ratio (CCR). Here, CCR is defined as correctly classified samples versus all the samples.
\end{abstract}

\section{General Terms}

Biological Image Processing.

\section{Keywords}

Classification, Segmentation, Computer-aided detection (CAD), Spinal Tuberculosis (TB).

\section{INTRODUCTION}

The textures are usually considered as intensity variations in the image. Since the intensity can vary in numerous ways, defining texture is not easy. However, the job of defining the texture can be dealt with when it is spread over a constant background. Such problems have been investigated in the past for various applications related to segmentation, for example, object recognition, tracking, and machine vision [1-3].

The task of detection of suspicious lesions of radiological images is very challenging because of numerous factors. These factors are influenced by the variation of intensity during the image acquisition, patient's anatomy inside as well as outside the field of interest, varying distance between the object and the film, focusing, noise, etc. Although a trained person overrides these factors in the visual diagnosis that he engages in, they greatly affect the performance of automatic segmentation and classification process [4]. Hence, even today, most of the clustered cellular level deterioration is heuristically estimated using the human expertise, as generally done by a radiologist. Several approaches have been proposed for achieving CAD of diseases through radiological images [4-12], but they were developed and tested under mutually different conditions. It is not possible to compare the effectiveness of these approaches as they are evaluated over varying data sets in different conditions.

Imaging for spinal TB is a regular radiographic exercise. But realizing a proper computer-aided diagnostic around the radiology image is remaining a challenge. The domain of spine images has been sparingly explored for CAD due to the inherent inhomogeneity of the spine structures and images, and the variety of challenges associated with automatic classifications [4].

The authors are not working in the domain of clinical proof generation and intend to provide the technology for identifying suspicious lesions that are mainly caused by TB occurrence through digital X-rays of the spine.

\section{METHODOLOGY}

A general CAD system framework is shown in Figure 1. The system comprises of six modules. First, a digital X-ray (DXR) undergoes the preprocessing steps that involve image enhancement and noise removal. In the next step, Regions Of Interest (ROIs) are cropped with the help of two professional radiologists. The relevant features are identified and extracted from the candidates in step 3. Finally, an SVM classifier is applied to train and test the candidates. The validation of the proposed technique is done at two levels: local and global. In the case of global testing, a correction step is also applied to the classification results.

\section{GENERATION OF THE DATASET}

The images (TB and normal spine) are collected from IMS BHU, Varanasi, and Arihant Diagnostics, Varanasi.

The digital X-ray images are in Dicom format. The area of the detector used is $3200 * 4200$ pixels, and size of a pixel is 139 micrometers. All images are enhanced by mapping the gray values such that $1 \%$ of data are saturated at low and high intensities. To sharpen the features and flatten the lighting variations in the image, a homomorphic wavelet filter with a double-level decomposition is applied. The ROI of size $128 * 128$ pixels are cropped covering TB affected inhomogeneous regions, and saved in a dataset named 'TB'. Normal regions of the healthy spine X-ray images were also cropped to the same size and stored in a dataset named 'NOTB'. All the images are cropped under the supervision of a qualified doctor. A mask is created to remove unwanted areas automatically from the spine X-ray images. 


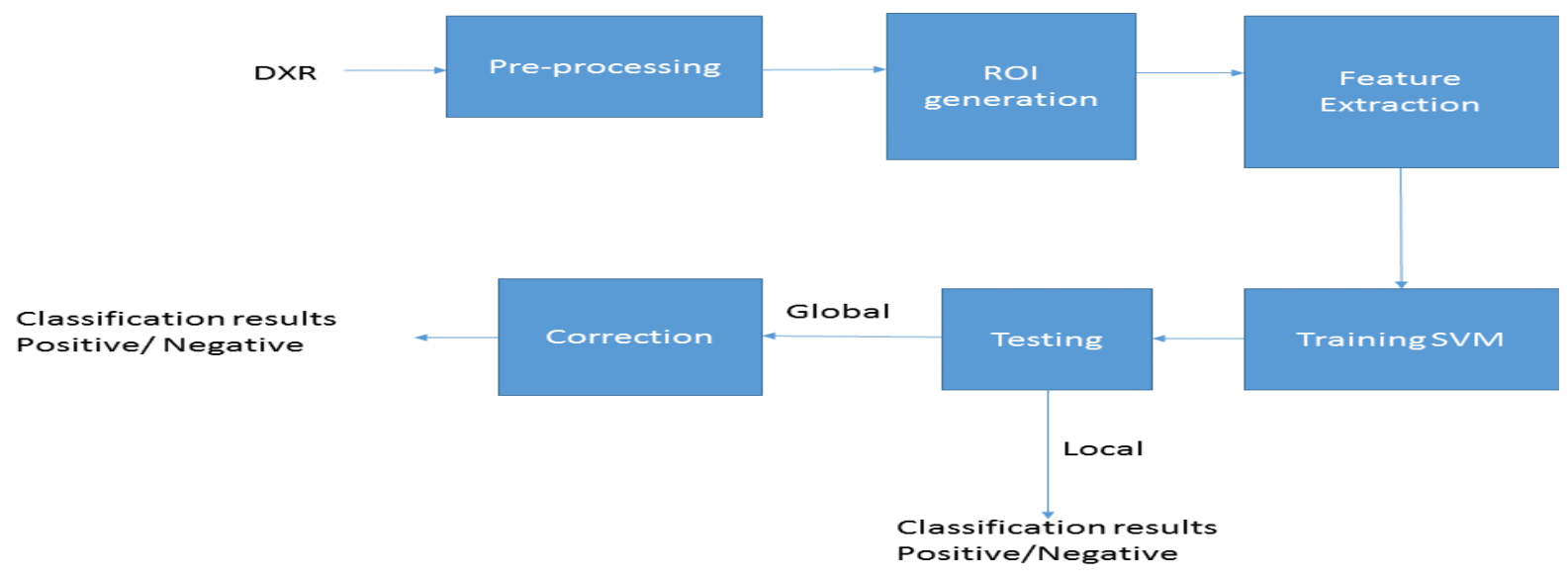

Fig 1: The processing steps of a CAD system for spine radiographs

\subsection{Generation of the mask}

During the period of study, through visual inspection of the spinal X-ray images, the authors found that looks brighter (higher in intensity) in comparison to the rest of the parts of the DXR. The authors used these observations to generate a method to remove unwanted areas automatically from the images. For similar cases, in the past, images are cropped manually [12]. In this method, first, the images were convolved with a mean window of size $401 * 401$ pixels shifting by one pixel at a time. The dimension of mean window has been chosen to be larger than the largest disk within the sample. The pixel at the center point of the window was replaced with the mean of all the $401 * 401$ points lying under the window. This process generated a mean image (Fig. 2(a)). In the next step, the mean image was further read row by row, and maxima of each row were plotted in red color (Fig. 2(a)). This red line was used as the spine line for generation of the mask (Fig. 2(b)). In order to discard the unwanted area around the spinal cord, 500 pixels to the left and right side of the maxima (keeping maxima in the middle) were retained for further analysis for each row and the rest were masked to zero (Fig. 2(c)). The window size of 500 pixels was chosen empirically to discard most of the unwanted areas.

Although the spinal cord is usually the brightest in a given window, this may not be necessarily the case in few rows. However, it does not affect the final diagnosis because such rows belong to the healthy spine as TB-affected areas look brighter in the X-ray. Losing some part of the healthy spine doesn't change the diagnosis.

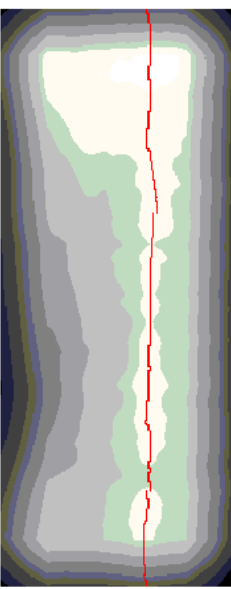

(a) Mean Image

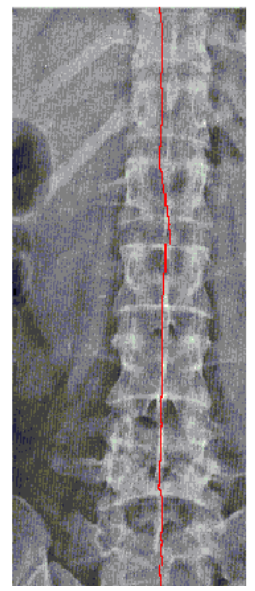

(b) Image with

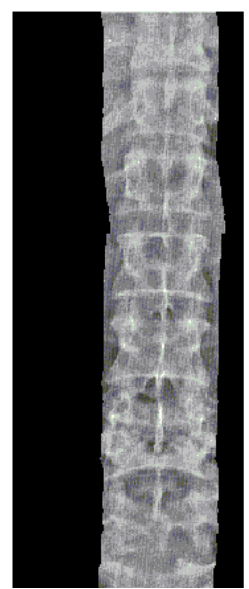

(c) Masked Image
Spine Linein red

Fig 2: Generation of the spine line and the masked image

\section{GENERATION OF THE FEATURE VECTOR}

Any feature extraction technique for machine learning is based on extracting common properties of the samples within a class, and eliminating common properties between the classes. This reduces the computation time required for classification and improves the classification accuracy. In order to identify the suitable set of feature descriptors, the following approach was used.

The initial Feature Vector (FV) was formed with eight basic texture features of the ROI. These eight texture features are: average gray level, average contrast, measure of smoothness, third moment, measure of uniformity, entropy, energy, and maximum column sum energy [5-7]. 


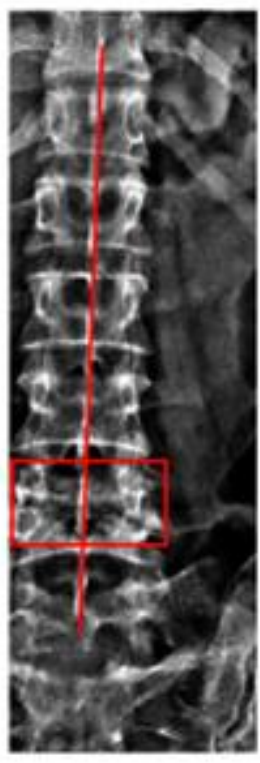

Fig 3: DXR of Healthy vertebrae and TB-affected vertebrae (in the red box)

The FV was further appended with more fine texture descriptors received from the Gray Level Difference Matrix (GLDM) [5]. In GLDM, difference images are created in four directions north east, north west, south east, and south west (theta $=0,45,90$ and 135 degrees respectively). The gray level histograms of these four new difference images are linearized. A systematic way was applied to plot and analyse the probability distribution function (pdf) of these histograms.

The authors observed that pdf with theta $=90$ degrees gave good descriptors between normal and abnormal cases. This indicates that the probability of gray-level distribution of the pair of pixels in the direction theta ( 90 degrees) are different for TB-affected ROIs and Normal ROIs. This finding can be appreciated/approved by sheer observation of Fig. 3; the difference between the clear horizontal boundaries of the healthy vertebrae and the unclear-hazy boundaries of the TBaffected vertebrae (in the red box) is maximum when viewed along the vertical line (theta $=90$ degree). Hence, GLDM of the image with theta $=90$ degree was also added to the FV; this gave us a more appropriate feature vector (FV1).

The lines/edges in images are formed by pixels with very high/low magnitude as compared to the environment, and Gabor filter is well-known for edge detection [9]. Extensive work done in the direction of Gabor filters with X-ray images gave the leads that log-Gabor filters [10], tuned with specific parametric values, can be utilized to extract unclear edges/boundaries in spine X-ray images. Analysing images at the intensity level have limitations as the phase property of interlinked pixels of a cluster in a region cannot be accurately detected and estimated $[1,11]$. To cater to the need to extract complete information from an image, it is necessary and recommended to deal with the frequencies involved in it along with phase variations $[8,11]$. Hence, Direction Density Matrix (DDM) of the Gabor phase plot is introduced as a feature descriptor in our work.

\subsection{DDM of the Gabor phase plot}

In this process, the ROI is filtered with Log-Gabor filter [10] $(\mathrm{f} 0=2.27, \sigma$ on $\mathrm{f}=0.5)$ for $\mathrm{N}$ different orientations $(\mathrm{N}=30)$.

The authors made a DDM to store all thirty responses produced by the filter. The DDM is defined as a matrix of size $128 * 128 * 30$. Each point in the $\operatorname{DDM}(\mathrm{x}, \mathrm{y}, \mathrm{n})$ represents the Gabor filter response at point (x,y) in nth orientation (Fig. 4).

Two plots of DDM is generated: Amplitude plot and Phase plot. The Amplitude plot is a matrix of size same as the input image (128*128), that contains the maximum magnitude at each point out of ' $N$ ' Gabor responses. The Phase plot records angles for which the magnitude of amplitudes was maximum.

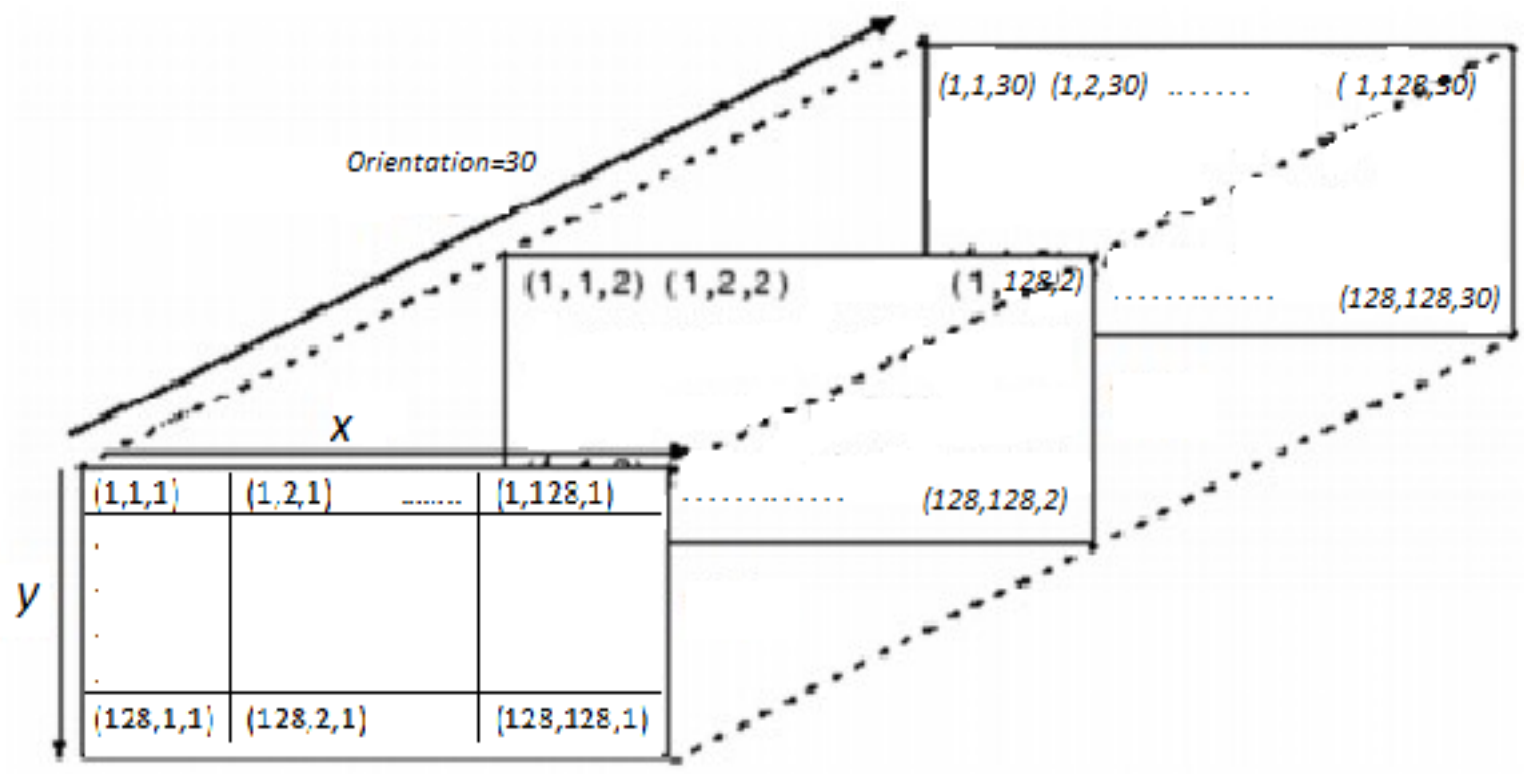

Fig 4: Structure of Direction Density Matrix 
Thus, each element in the phase map is valued between 1 and $\mathrm{N}$. This kind of feature combines the position information and orientation information.

$$
\text { Amplitude } \operatorname{plot}(x, y)=\operatorname{Max}(\operatorname{DDM}(x, y, n))_{\{\mathrm{n}=1: N\}}
$$

Phase plot $(x, y)=\operatorname{argmax}_{n}(\operatorname{DDM}(x, y, n))$

To incorporate the phase property into the feature vector and ensure the preservation of textural details, GLDM of Phase plot with theta $=135$ degrees was used as one of the feature descriptors.

As the magnitude of the spectrum contains more information related to the overall look of the image rather fine details, a summed up value of the Amplitude plot is sufficient as a feature. Hence, the Amplitude plot of the orientation map was summed up (Asum) and taken as a descriptor. The FV1 was further appended with descriptors received from phase and amplitude plot (as explained above) to generate FV2.

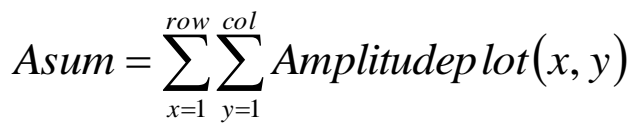

5. EXPERIMENTATION AND RESULTS

The validation of the proposed technique has been done at two levels: local and global.

\section{Local Method}

This method uses ROIs of size $128 * 128$, which are cropped manually and kept in databases TB and NOTB (as explained in section 3) for testing. After appropriate scaling, feature sets (FV1 and FV2) were generated for each image ROI. The authors evaluated the proposed technique using $32 \mathrm{X}$-ray images (16 TB+16 NOTB) of the spine, randomly selected from a database of 50 images. Eight TB and eight NOTB images were randomly selected from sixteen TB and sixteen NOTB images, and used for training the SVM classifier (box constraint $\mathrm{C}=3$ ). Feature vectors extracted from the other eight TB and eight NOTB images, which were not used for training, were used for testing. The performance measures used are Correct Classification Ratio(CCR), Sensitivity, and Specificity. CCR gives correctly classified samples (CCS) divided by the classified samples (CS). Sensitivity can be defined as correctly classified positive samples (CCP) divided by true positive (TP) samples. Specificity stands for correctly classified negative samples (CCN) divided by the true

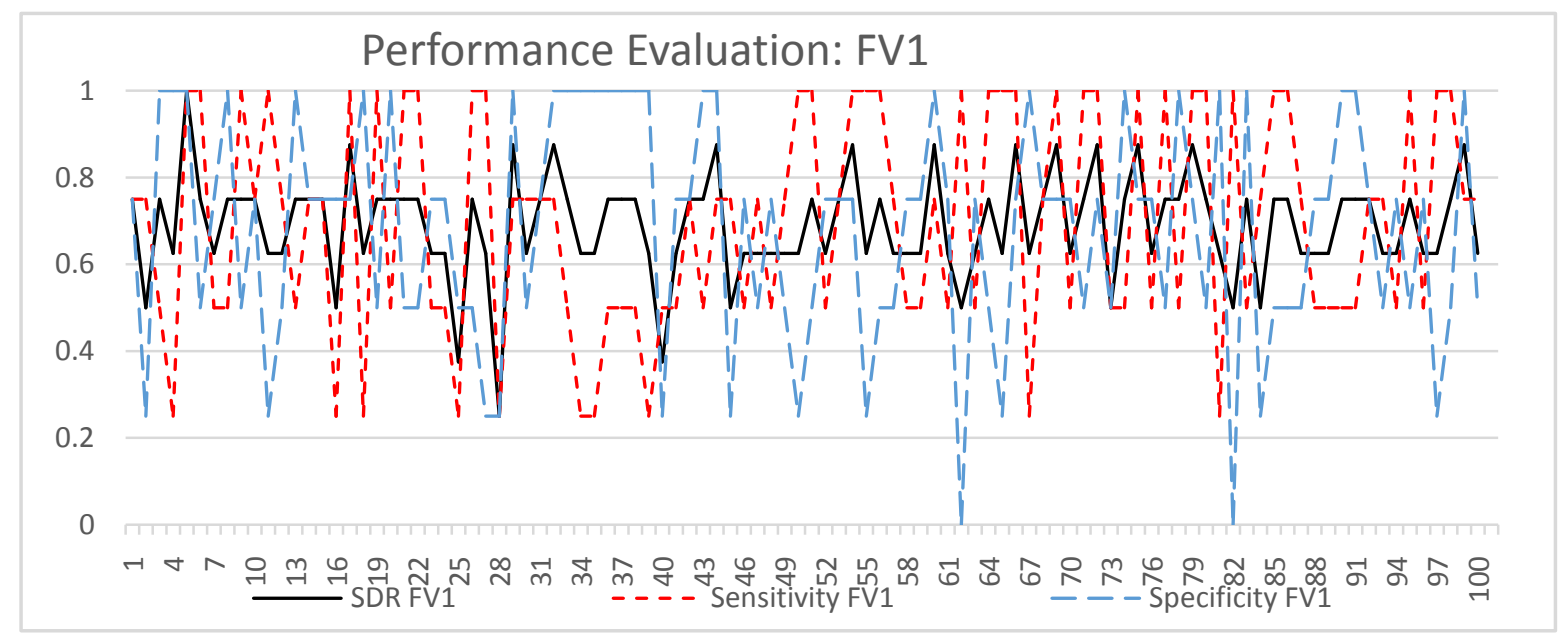

(a)

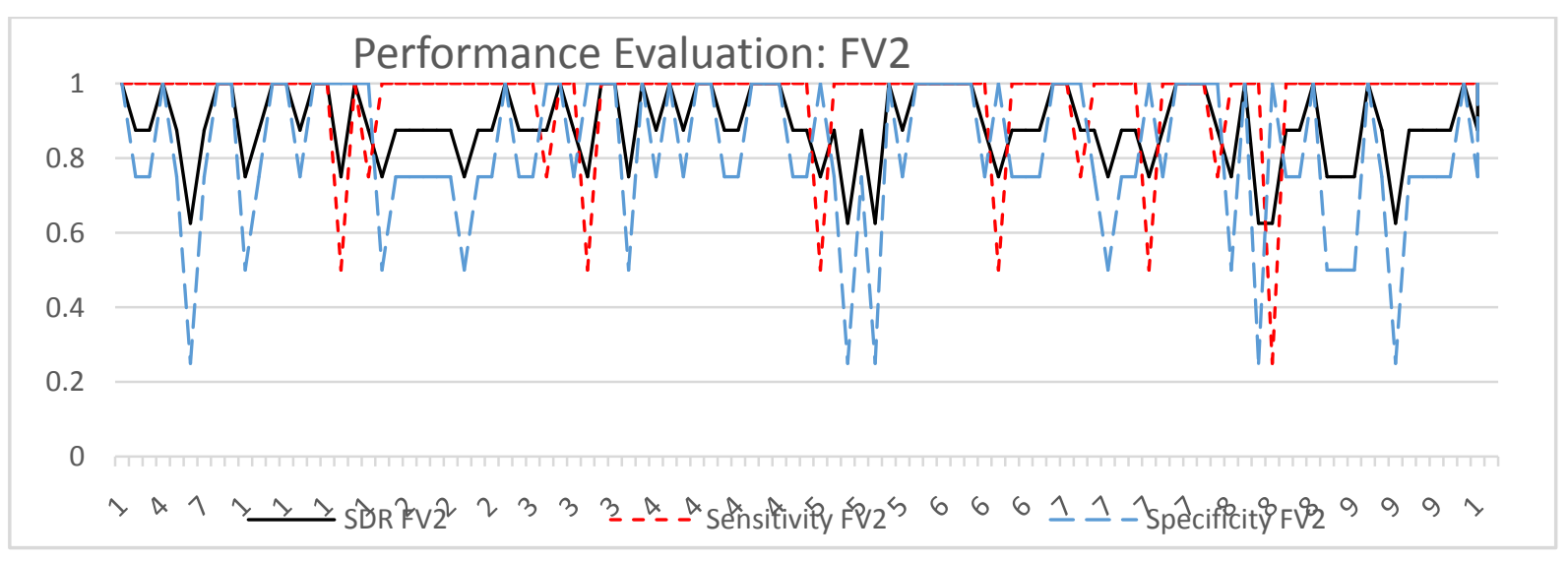

(b)

Fig 5: Performance evaluation for 100 iterations (a) FV1 (b) FV2 
Table 1. Mean Performance Evaluation: Local Method

\begin{tabular}{|c|c|c|c|}
\hline Feature Set & Mean Correct Classification Ratio & Mean Sensitivity & Mean Specificity \\
\hline FV1( Basic+ GLDM of the image) & 0.69 & 0.6975 & 0.6825 \\
\hline FV2( FV1 + GLDM of phase plot+ Asum) & 0.9437 & 0.9675 & 0.9125 \\
\hline
\end{tabular}

negative samples $(\mathrm{TN})$.

The training and testing procedure are repeated 100 times, and the performance measurements are tracked in every iteration (Fig. 5). The variation in performance for each iteration is due to the set of images randomly selected for training and testing. The final results are the average performance of the 100 runs (Table 1).

Mean Correct Classification Ratio $(\mathrm{CCR})=$

$$
1 / 100\left[\sum_{i=1}^{100}\left(\frac{C C S(i)}{C S(i)}\right)\right]
$$

Mean Sensitivity $=1 / 100\left[\sum_{i=1}^{100}\left(\frac{C C P(i)}{T P(i)}\right)\right]$

Mean Specificity $=1 / 100\left[\sum_{i=1}^{100}\left(\frac{C C N(i)}{T N(i)}\right)\right]$

The graphs depict the classification results with two different feature sets (Fig. 5). CCR can have a maximum value of one and a minimum value of zero. If the value of CCR is one, it means that all the images in the database are classified correctly, and zero value indicates that all the images are classified incorrectly. It is evident from the graphs that CCR with FV2 looks impressive in most of the iterations with its value lying between 0.9 and 1.0 (Fig. 5). Once the CCR is between 0.9 and 1.0, it means that $90 \%$ to $100 \%$ images are classified correctly in most of the iterations.

\section{Global Method}

The local method of validation uses ROIs, which are cropped

manually for training as well as for testing. In this approach, the rest of the image area, which is not part of the ROI, goes invalidated. The global method uses 50 uncropped X-ray images of the spine for testing. In this method, first, a mask is applied to each image as explained in section 3.1. Next, the masked image is cut into non-overlapping windows of size $128 * 128$. Each image produces 114 such windows. A Feature Vector (FV2) is generated from each window and is used for testing with SVM, which was trained from 49 ROIs collected from the TB and NOTB databases (excluding the ROI, which is cropped from the image used for testing).

The image-wise performance evaluation using the global method over 6 test cases is shown in Table 1. It can be seen that false positives are present in almost all the images. This happens because the window of $128 * 128$ is too small in size, so sometimes a window in healthy images may depict the features of TB (hazy boundaries) and can be misclassified. The authors included a correction step after classification to correct such false positive cases. Since TB-affected areas are generally much bigger than $128 * 128$ pixels in our radiographs, in the correction step, isolated marked blocks (an abnormal block with all 8 neighbours as normal) are removed before classifying overall image and such images are finally classified as 'normal'. Admittedly, this way it is possible to miss classifying TB-affected areas of single window size. But it is not of much concern because, TB affected vertebrae show hazy boundaries bigger than a double window size for

One of the major findings of this research is that the abnormality detection capability of spinal cord aberrations can be achieved by introducing innovative features generated by DDM of the phase map.

The authors tested the effectiveness of the circular profile of FFT, as suggested in [13]. It was found that using the circular profile of FFT in the feature vector does not help in improving the classification accuracy in our case.

The authors also compared their results with other wellestablished features, such as local binary patterns (LBP). The LBP coded image and the local binary pattern histogram of the image blocks were used as features and a variety of feature vectors were generated using them as suggested in [14] but they could give the accuracy in a range of $80-85 \%$ only. The proposed method gives a mean CCR of $94.37 \%$ with just 21 points feature set.

\section{CONCLUSIONS}

A phase-sensitive feature set has been developed for improving the classification capability of suspicious lesions in diffused texture images as encountered in biomedical scenarios. An efficient technique has been developed to mask the area around the spinal cord automatically in digital X-ray images of the spine. To verify the effect of phase-sensitive parameters, the authors used the phase plot of the DDM as a part of the feature set. Experimental results have shown that use of phase-sensitive parameters increases the efficacy of the classification process and helps in improving the classification accuracy substantially $(25.37 \%)$.

Though images with a frontal view of the spine were used for this analysis, the transverse view along with the frontal view also can be used in the future for improved results.

\section{REFERENCES}

[1] Greef MD, Weve R, Kerkstra S, Tracking Objects using Gabor Filters, Profile Project 2006

[2] Gonzalez RC, Woods, Eddins, Digital image processing using MATLAB. Object Recognition. 2nd ed., Delhi, India, Pearson Education, 2nd ed., 498-522, 2009

[3] Collins RT, Liu Y, Leordeanu M, Online Selection of discriminating Tracking Features. IEEE Transaction on Pattern Analysis and Machine Intelligence, Vol 27, No. 10, 1631-1643 (Oct.2005)

[4] Diniz PR, Murta-Junior LO, Brum DG, de Araújo DB, Santos AC, Brain tissue segmentation using q entropy, Brazilian Journal of Medical, 43(1),77-84 ( 2010), 77-84, http://dx.doi.org/10.1590/S0100-879X2009007500019 
[5] Kim JK, Park HW, Statistical texture features for detection of microcalcifications in Digitized Mammograms. IEEE Transactions on medical imaging, Vol 18, No. 3, 231-238 (1999)

[6] Noor NM, Rejal OM, Yunus A, et al. A statistical interpretation of the chest radiograph for the detection of pulmonary tuberculosis. Conference on Biomedical Engineering and Sciences (IECBES), Kuala Lumpur,2010 IEEE EMBS, 47-51

[7] Patil SA, Udupi VR, Kane CD, Wasif AI, Geometrical and texture features estimation of lung cancer and TB images using chest X-ray database, International Conference on Biomedical and Pharmaceutical Engineering - ICBPE, 2009. doi: 10.1109/ICBPE.2009.5384113

[8] Steven W. Smith, The Scientist, and Engineer's Guide to Digital Signal Processing, California Technical Publishing, ch. 13, 567-578, 2011.

[9] Z. Xiao, C. Guo, Y. Ming, and L. Qiang, Research on log Gabor wavelet and its application in image edge detection, International Conference on Signal Processing, Aug 2002, vol. 1, 592-595
[10] Daugman J, New methods in iris recognition. IEEE Trans. Systems, Man, Cybernetics B 37(5), 1167-1175 (2007).

[11] T.R.Gopalakrishnan Nair, Richa Sharma, Pre transplant visualization of combined images for predictive medical analysis, Journal of Medical Engineering and Technology, 38(4),220-226, (2014). doi:10.3109/03091902.2014.904015

[12] Michopoulou, S.; (2011) Image analysis for the diagnosis of MR images of the lumbar spine. Doctoral thesis, pp. 80, UCL (University College London)

[13] Jennifer S Gregory, Alison Stewart, Peter E Undrill,, David M Reid and Richard M Aspden, "Identification of hip fracture patients from radiographs using fourier analysis of the trabecular structure: a cross-sectional study”, BMC medical imaging, 2004

[14] Ojala T, Pietikäinen M, Mäenpää T, "Multiresolution gray-scale and rotation invariant texture classification with Local Binary Patterns". IEEE Transaction on pattern analysis 2002 